\title{
Effect of ultrasound-guided interstitial laser photocoagulation on benign solitary solid cold thyroid nodules - a randomised study
}

\author{
Helle Døssing, Finn Noe Bennedbæk ${ }^{1}$ and Laszlo Hegedüs ${ }^{1}$ \\ Departments of Oto-rhino-laryngology and ${ }^{1}$ Endocrinology and Metabolism, Odense University Hospital, Odense, Denmark
}

(Correspondence should be addressed to H Døssing, Department of Oto-rhino-laryngology, Odense University Hospital, DK-500O Odense C, Denmark; Email: helle.doessing@ouh.fyns-amt.dk)

\begin{abstract}
Aim: To evaluate the efficacy of ultrasound (US)-guided interstitial laser photocoagulation (ILP) on thyroid function, nodule size and patient satisfaction in benign solitary solid cold thyroid nodules by comparing one ILP session with no treatment in a prospective randomised study.

Materials and methods: Thirty euthyroid outpatients with a benign solitary solid and a scintigraphically cold thyroid nodule causing local discomfort were assigned to one session of ILP $(n=15)$ or observation $(n=15)$ and followed for 6 months. Thyroid nodule volume and total thyroid volume were assessed by US and thyroid function was determined by routine assays before and during follow-up. Pressure and cosmetic complaints before and at 6 months were evaluated on a visual analogue scale. ILP was performed under US guidance and with an output power of $2.5-3.5 \mathrm{~W}$.

Results: In the ILP group, the nodule volume decreased from $8.2 \mathrm{ml}(6.1 ; 11.9)$ (median; quartiles) to $4.8 \mathrm{ml}(3.0 ; 6.6)$ after 6 months $(P=0.001)$. The overall median reduction was $44 \%(37 ; 52)$, which correlated with a significant decrease in pressure symptoms as well as cosmetic complaints. In the control group, a non-significant increase in median nodule volume of $7 \%(0 ; 34)$ after 6 months was seen. No major side-effects were seen in the ILP group. There was no correlation between thermal energy deposition and nodule volume reduction. Thyroid function was unaltered throughout.

Conclusion: US-guided ILP, given as a single treatment, resulted in a satisfactory clinical response in the majority of patients with a benign solitary solid cold thyroid nodule, and may become a clinically relevant alternative to surgery in selected patients.
\end{abstract}

European Journal of Endocrinology 152 341-345

\section{Introduction}

Nodular goitre is common in the adult population and the prevalence increases with age $(1,2)$. Most thyroid nodules are benign. However, the natural history is poorly understood, although it seems that approximately $20 \%$ or more of the solid nodules increase in size over time $(3,4)$. While nodule growth is often regarded as suspicious for malignancy, data on this issue are limited and inconsistent (3). Thorough clinical and biochemical evaluation and ultrasound (US)-guided fine-needle aspiration biopsy (FNAB) of solitary thyroid nodules reduce the risk of overlooking clinically relevant malignancy to no more than $1 \%$ (5). The use of USguided FNAB seems to reduce the number of diagnostic thyroidectomies (6) and thereby allows a non-surgical approach in patients with symptoms of compression (1). The therapeutic strategy for the euthyroid patient with a benign solitary thyroid nodule that appears cold on a radionuclide scan is still a matter of debate (1). The possibilities are surveillance, L-thyroxine (L-T4) suppression therapy, percutaneous ethanol injection (PEI) or interstitial laser photocoagulation (ILP) (5). L-T4 treatment has little or no effect on nodule size and symptoms $(5,7)$. US-guided PEI has shown to be effective in inducing necrosis $(7,8)$. The limitations of PEI are the difficulty in predicting the diffusion of the ethanol which can cause pronounced pain, and sideeffects such as extraglandular fibrosis impeding subsequent surgery, needed in case of treatment failure. This clearly limits the use of PEI and explains our reluctance to introduce this treatment for routine use in solid nodules. However, in benign solitary cystic nodules PEI performs well with few side-effects (9). Using low power laser energy, the necrosis induced by the thermic energy can be delivered in a controlled fashion with no or only minimal damage to the surrounding tissue (10). Therefore, ILP has been introduced for ablation of both malignant and benign lesions $(4,11-18)$. The technique has been introduced in non-randomised feasibility studies treating patients with benign solid thyroid nodules $(4,19-21)$. ILP was well tolerated and the side-effect reported was slight discomfort or transient pain. In one study, transient 
vocal cord paresis was recorded in one patient (20). Our aim was to evaluate the effect of US-guided ILP on the volume of benign solitary solid cold thyroid nodules as well as nodule-related symptoms and thyroid function in a prospective randomised study. The purpose was to confirm our previous results from a feasibility study and to exclude an effect due to spontaneous nodule reduction by having used a comparable parallel group of untreated patients.

\section{Materials and methods}

Patients were referred from their primary care physicians because of a palpable thyroid nodule. All patients had a solitary cold solid benign thyroid nodule evaluated clinically, scintigraphically and by US-guided FNAB. Cytology was compatible with a colloid nodule. All patients reported cosmetic and/or pressure symptoms. None had suspicion of or a family history of thyroid cancer. None had had prior radiation to the neck and patients were between 20 and 70 years. All patients were euthyroid and had normal serum calcitonin levels. Blood tests included serum thyrotrophin (TSH; normal range, $0.3-4.0 \mathrm{mU} / \mathrm{ml}$ ) determined by DELFIA (Wallac, Oy, Turku, Finland), serum total T4 (normal range, 65-135 mmol/l) determined by RIA (Diagnostic Products Corp., Los Angeles, CA, USA) and serum total tri-iodothyronine (T3; normal range, 1.00-2.10 mmol/l) determined by RIA (Johnson \& Johnson, Clinical Diagnostics Ltd, Amersham, Bucks, UK). Free T4 and T3 (FT4 and FT3) indices were calculated by multiplying serum $\mathrm{T} 4$ and $\mathrm{T} 3$ levels by the percent T3 resin uptake. Serum antithyroid peroxidase antibodies (anti-TPOAb) were determined by RIA DYNO test (Brahms Diagnostica GmbH, Berlin, Germany; normal range $<60 \mathrm{U} / \mathrm{ml})$. All patients were offered standard therapy (surgery) but declined and they were randomised consecutively. Random allocation was achieved using a random number generator on a computer. Patients were assigned to (i) one treatment session of US-guided ILP or (ii) observation for 6 months. At enrolment and at the 6-month evaluation the patients were asked to rate pressure symptoms and cosmetic complaints on a visual $10 \mathrm{~cm}$ analogue scale $(0-10 \mathrm{~cm})$. In patients treated with ILP, an indirect laryngoscopy was performed immediately before and 6 months after treatment by a specialist in otorhino-laryngology to evaluate the motility of the vocal cords. ILP was carried out on an outpatient basis. Immediately after termination of the ILP procedure the patients were asked to rate the degree of pain/discomfort on a visual analogue scale and whether they would undergo the treatment again, as a surrogate marker of tolerability. The patients were investigated 1,2 and 6 months after the treatment. Initially, and at the follow-up, nodule volume and thyroid volume as well as serum thyroid hormones and serum TSH were investigated. Furthermore, anti-TPOAb were measured at the 6-month evaluation.

A Logiq 500 US scanner (GE Medical Systems, Milwaukee, WI, USA) with a $12 \mathrm{MHz}$ linear transducer (type 739L) mounted with a needle-steering device for precise US-guided punctures was used. Under sterile conditions and guided by US, the laser fibre $(0.4 \mathrm{~mm}$ in diameter) was positioned in the thyroid nodule through the lumen of an 18 gauge $(1.2 \mathrm{~mm})$ needle and preceded by local anaesthesia with lidocaine $(10 \mathrm{mg} / \mathrm{ml})$. The needle was withdrawn $20 \mathrm{~mm}$ leaving the end of the fibre in direct contact with the tissue. Patients were then treated with an output power of 2.5$3.5 \mathrm{~W}$, dependent on pretreatment nodule volume and the position of the nodule. The entire procedure was performed under continuous US guidance with an infra-red diode (model 15; Diomed, Cambridge, UK) laser power source. During laser treatment the vapor from the tissue was clearly visible on US as an irregular echogenic area enlarging over time. Based on the experience of Pacella et al. (16), at present we suggest a safety distance from the neurovascular bundle of at least $1.5 \mathrm{~cm}$ to avoid injury to these structures as a result of the thermal effect of ILP. Typically, three or four areas were treated before the procedure was terminated. The energy delivered during photocoagulation was recorded. Thyroid nodule volume was calculated on the basis of an ultrasonic scanning procedure, by recording cross-sections through the gland, using a $5.5 \mathrm{MHz}$ static compound scanner (type 1846; B\&K Medical, Gentofte, Denmark). The measurements were done by the same investigator (H D), as previously described (7), and with the investigator blinded as to previous results and with an intra- and inter-observer variation of $5-7 \%(7)$. The protocol was approved by the ethics committee of the county of Funen. Prior to participation the patients all gave signed informed consent.

\section{Statistics}

We know from a previous study (4) that the standard deviation on proportional nodule volume reduction was $5.1 \mathrm{ml}$. Given a type I error of 0.05 (two-sided), a power of $80 \%$ and a clinically relevant difference of $5 \mathrm{ml}$ gives a sample size of 15 patients in each group. Results for continuous data are given as median and quartiles. All comparisons for paired data in the two groups are based on ANOVA II (observation based on within-subject difference; serial measurements; TSH and FT4 and FT3 index). Nodule volume change in the treatment group and control group was tested by means of a Wilcoxon signed ranks test. Comparison of initial thyroid nodule volume between the two groups was performed by a Mann-Whitney test. Correlation between energy deposition and reduction in nodule volume at the 6-month evaluation was performed by means of Spearman's rank correlation coefficient. 
Pressure symptoms and cosmetic complaints in the treatment group were rated on a $0-10 \mathrm{~cm}$ visual analogue scale before and 6 months after treatment and changes were evaluated by a sign test.

\section{Results}

Clinical data of the 30 consecutive patients randomly assigned to observation or ILP treatment are given in Table 1. The groups were similar with regard to age, sex, nodule duration, nodule volume, thyroid volume, TSH, FT4 index, FT3 index, serum calcitonin level and number of cases with anti-TPO antibodies. All patients had low or no uptake in the nodule on $\left[{ }^{99} \mathrm{~m}\right.$ Tc]pertechnetate scintigraphy and had a solitary and solid nodule on US. US-guided cytology was benign in all subjects.

Four of the patients had had a previous partial thyroidectomy for benign nodular goitre. In the control group the median nodule volume increased from $7.5 \mathrm{ml}(5.1 ; 13.8$ (quartiles)) to $9.0 \mathrm{ml}(5.0 ; 14.0)$ $(P=0.07)$ at 6 months, i.e. a median increase of $7 \%$ $(0 ; 34)$. Total thyroid volume was unchanged during follow-up $(P=0.9)$. There was no change in pressure symptoms $(P=1.0)$ or cosmetic complaints in this group $(P=1.0)$ (Fig. 1$)$. There were no significant changes in serum TSH $(P=0.2)$, FT4 index $(P=0.2)$, FT3 index $(P=0.2)$ or anti-TPOAb levels $(P=1.0)$ during follow-up (data not given). In the ILP group, the median nodule volume decreased from $8.2 \mathrm{ml}(6.1 ; 11.9$ (quartiles)) to $6.1 \mathrm{ml}(3.7 ; 9.3)$ after 1 month $(P=0.0002)$ and $4.8 \mathrm{ml}(3.0 ; 6.6)$ after 6 months $(P=0.001)$ (Fig. 2). The overall median reduction at 6 months was $44 \%(37 ; 57)$ and the total thyroid volume decreased from $26.0 \mathrm{ml}$ (18.6; $31.0)$ to $24.1 \mathrm{ml}(20.3 ; 31.6) \quad(P=0.005)$. There were no overall changes in serum TSH $(P=0.4)$, FT4 index $(P=0.9)$ or FT3 index $(P=0.3)$ levels during follow-up. Three patients had an unaltered low value of anti-TPOAb $(P=1.0)$ during follow-up. One patient was subsequently operated on because of an unsatisfactory result. Histology demonstrated a benign colloid nodule. Initially, 13 of 15 patients in the ILP group had pressure symptoms. At the 6-month follow-up ten patients had marked relief of pressure symptoms while three patients experienced no relief. One patient reported aggravation in pressure symptoms (pretreatment score of 5 and a score of 10 at 6 months; visual analogue scale) although a reduction in nodule volume of $26 \%$ was seen. This patient had a fracture of the clavicula and the aggravation of the neck symptoms were most likely related to this, since her symptoms disappeared a few months later. Both the cosmetic complaints $(P=0.02)$ and the pressure symptoms $(P=0.04)$ were significantly reduced during follow-up (Fig. 1).The only side-effect due to ILP was slight to moderate pain in seven patients lasting for up to 3 days. Three patients experienced tenderness for up to 1 week. The treatment was well tolerated as evidenced by the fact that none of the patients asked at the termination of the procedure - would decline a new ILP treatment if offered later. No serious side-effects such as dysphonia, infection, haematoma, bleeding, hyperthyroid symptoms or vocal cord paralysis were encountered.

The median total energy deposition was 2007 J (1750; 2880 (quartiles)). The median energy given per $\mathrm{ml}$ of the pretreatment nodule volume was 224J (182; 331 (quartiles)). When the area was stationary in size, typically after 360-540 J, the fibre was replaced into another area in the nodule and this procedure was performed until all accessible parts of the nodule had been treated. There was no significant correlation between nodule volume reduction and energy deposition $(r=0.2 ; P=0.4)$.

\section{Discussion}

Based on the fact that only a minority of benign solitary solid and cold thyroid nodules will decrease spontaneously over time, many patients are offered surgery in case of compressive symptoms (1). Several studies have confirmed that PEI is effective in inducing necrosis and subsequent nodule shrinkage up to 1 year after treatment $(7,8,22,23)$. Although easily performed, PEI has limitations related to ethanol escaping outside the nodule capsule and even the thyroid $(5,8,23)$.

Table 1 Baseline characteristic of the two groups. Values are median (quartiles) or number of cases.

\begin{tabular}{|c|c|c|c|}
\hline Variable & Observation & Laser treatment & $\boldsymbol{P}$ \\
\hline Age (years) & $46(41 ; 51)$ & $47(43 ; 52)$ & 0.7 \\
\hline $\operatorname{Sex}(M / F)$ & $0 / 15$ & $0 / 15$ & 1.0 \\
\hline Months from diagnosis & $6(3 ; 7.5)$ & $6(3 ; 12)$ & 0.4 \\
\hline $\mathrm{TSH}(\mathrm{mU} / \mathrm{l})^{\mathrm{a}}$ & $0.97(0.84 ; 1.63)$ & $0.96(0.64 ; 1.80)$ & 0.7 \\
\hline FT4 index $(\mathrm{U} / \mathrm{l})^{\mathrm{b}}$ & $105(98 ; 122)$ & $108(85 ; 130)$ & 0.7 \\
\hline FT3 index $(U / I)^{c}$ & $1.7(1.5 ; 2.0)$ & $1.8(1.4 ; 1.9)$ & 0.5 \\
\hline Anti-TPOAb (negative/positive) & $13 / 2$ & $14 / 1$ & 0.6 \\
\hline
\end{tabular}

${ }^{\text {a }}$ TSH value: $0.3-4.0 \mathrm{mU} / \mathrm{l}$.

${ }^{\mathrm{b}} \mathrm{FT} 4$ index $=\mathrm{T} 4 \times \mathrm{T} 3$ resin uptake, normal value: $60-140 \mathrm{U} / \mathrm{l}$

${ }^{\mathrm{c}} \mathrm{FT} 3$ index $=\mathrm{T} 3 \times \mathrm{T} 3$ resin uptake, normal value: $0.95-2.20 \mathrm{U} / \mathrm{l}$. 

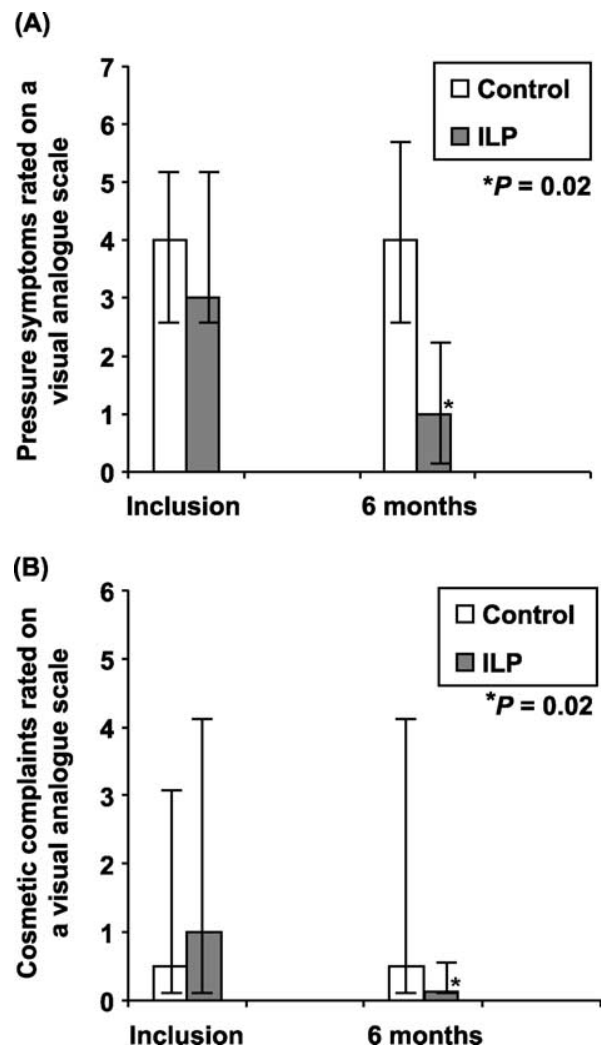

Figure 1 (A) Pressure symptoms and (B) cosmetic complaints (median and quartiles) evaluated by a visual analogue scale $(0-10 \mathrm{~cm})$, before and after 6 months of follow-up.

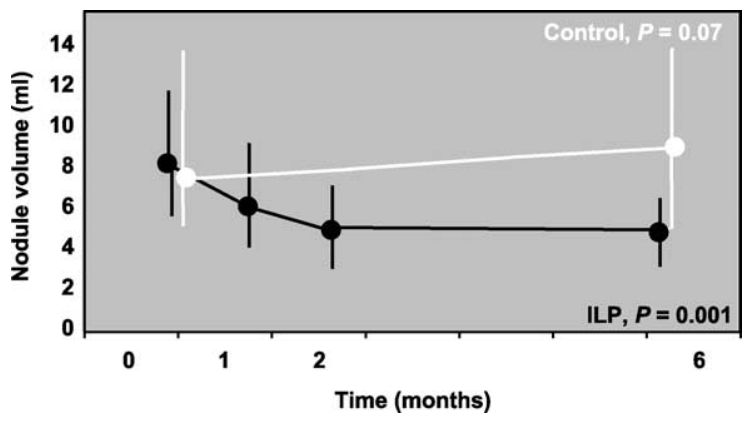

Figure 2 Thyroid nodule volume before and 1,2 and 6 months after ILP treatment and before and 6 months after observation (median and quartiles).

In contrast to ethanol, the laser-induced destruction zone and necrotic area can be controlled, resulting in no or minimal damage to the surrounding tissue (16). When monitored by US, the fibre tip is clearly visualised as a hyperechoic spot, and during ILP an irregular hyperechoic area enlarging over time is seen. This serves as a rough measure of the extent of the area of necrosis during the procedure. Differences in tissue characteristics might well account for the unpredictability of the effect of the laser treatment and an exact surrogate marker in addition to sonographic changes during treatment is needed. This might be one of the shortcomings of the treatment since the correlation between US findings and the extention of tissue necrosis is often unpredictable. The difficulty in visualising the exact extension of the treatment makes it impossible to perform a total ablation as contiguous structures (laryngeal nerve, carotid artery and upper airway) may be close to the nodule. Histological tissue examination after ILP confirms the presence of a welldefined necrotic area, demonstrating the ability of this technique to cause a coagulative necrosis in a controlled fashion $(16,24)$. In our study, median nodule volume reduction was $44 \%$ after 6 months following a single treatment session with ILP. This is similar to that which we have demonstrated in a previous nonrandomised feasibility study (4). As with PEI, most of the effect was evident within the first month and no significant reduction in nodule volume was seen during the remaining period from 1 to 6 months $(7,8)$. A logical endpoint of therapy, ignored in most clinical trials, should be relief of symptoms, defined by the patients, rather than a definite reduction in nodule volume. As a consequence, we designed a simple questionnaire to quantify the effects on pressure symptoms and cosmetic complaints. We found a significant correlation between relief of symptoms and nodule volume reduction in the ILP-treated group. These results were similar to that of our previous ILP study and that of PEI $(4,22)$.

With PEI as well as ILP there is no correlation between ethanol dose/ILP-energy deposition and reduction of the nodule volume $(4,8)$. In a small feasibility study comprising five patients with a scintigrafically cold thyroid nodule, Spiezia et al. (20) reported a nodule reduction of $61 \%$ following multiple sessions of ILP, and this result is confirmed in a recently published study (21). There are no discriminating features by US or pathology that can predict the outcome of ILP. Thermal necrosis at low energy occurs after several minutes. Power Doppler US during ILP may improve the outcome by aiding in the placement of the fibre and determining the length of the treatment depending on persistent vascularisation (20). However, surrounding vital structures adjacent to the nodule hinders treatment of certain areas. This will in many cases prevent total ablation of the nodule. Renewed growth may therefore be anticipated. Absolute temperature and duration of exposure are important parameters used to predict tissue damage. The threshold for irreversible tissue damage also depends on the type of tissue. As seen in the liver, there is a large degree of interindividual response to treatment (24). Most likely a number of factors such as pre-existing fibrosis, amount of colloid and fluid content of the nodules may account for the differences in outcome (8). Other biological characteristics, such as vascularisation of the nodule, are factors which can alter the thermal deposition of the laser energy (10). The endpoint, nodule volume reduction, is probably a two-step development after therapy. First, the thermal energy must destroy the tissue and then the lesion will shrink. This may, to 
some degree, explain the lack of correlation between nodule reduction and energy deposition. As in other studies, the ILP treatment was well tolerated by the patients $(4,15,16,18,20,21)$. However, mild local or radiating pain during ILP, which disappeared when the energy was turned off, and mild to moderate pain or tenderness lasting few days, was seen in half of the patients. A reason for more patients experiencing pain in the present study could be that the median energy used was $2007 \mathrm{~J}$ compared with $761 \mathrm{~J}$ in our previous study (4). In our borderline iodine-deficient area, the natural history is a slight increase in solitary nodule volume over time. The ILP procedure has proven to be a minimally invasive technique carried out on an outpatient basis and seems a promising non-surgical alternative, but further follow-up is needed to evaluate long-term efficacy. In non-randomised studies, repeated ILP treatments have been used with a higher degree of nodule reduction $(19,21)$. However, further ongoing randomised studies must address the efficacy of repeated treatments and the optimum energy deposition. ILP might become an alternative for highly selected patients, but the procedure should be restricted to experienced centres to avoid major complications.

\section{Acknowledgements}

This study was financially supported by the Agnes and Knut Mørk Foundation, the Novo Nordisk Foundation, the A P Møller Relief Foundation and the A J Andersen and Wife Foundation. We thank Annelise Krogdahl for her meticulous analysis of all the cytology specimens.

\section{References}

1 Hegedüs L. Clinical practice. The thyroid nodule. New England Journal of Medicine 2004351 1764-1771.

2 Singer PA, Cooper DS, Daniels GH, Ladenson PW, Greenspan FS, Levy EG, Braverman LE, Clark OH, McDougall IR, Ain KV \& Dorfman SG. Treatment guidelines for patients with thyroid nodules and well-differentiated thyroid cancer. American Thyroid Association. Archives of Internal Medicine $19961562165-2172$.

3 Alexander EK, Hurwitz S, Heering JP, Benson CB, Frates MC, Doubilet PM, Cibas ES, Larsen PR \& Marqusee E. Natural history of benign solid and cystic thyroid nodules. Annals of Internal Medicine 2003138 315-318.

4 Døssing H, Bennedbæk FN, Karstrup S \& Hegedüs L. Benign solitary solid cold thyroid nodules: US-guided interstitial laser photocoagulation - initial experience. Radiology $2002 \mathbf{2 2 5}$ $53-57$.

5 Hegedüs L, Bonnema SJ \& Bennedbæk FN. Management of simple nodular goiter: current status and future perspectives. Endocrine Reviews $200324102-132$.

6 Alexander EK, Heering JP, Benson CB, Frates MC, Doubilet PM, Cibas ES \& Marqusee E. Assessment of nondiagnostic ultrasound-guided fine needle aspirations of thyroid nodules. Journal of Clinical Endocrinology and Metabolism $2002874924-4927$.

7 Bennedbæk FN, Nielsen LK \& Hegedüs L. Effect of percutaneous ethanol injection therapy versus suppressive doses of L-thyroxine on benign solitary solid cold thyroid nodules: a randomized trial. Journal of Clinical Endocrinology and Metabolism $1998 \mathbf{8 3}$ $830-835$.
8 Bennedbæk FN \& Hegedüs L. Percutaneous ethanol injection therapy in benign solitary solid cold thyroid nodules: a randomized trial comparing one injection with three injections. Thyroid $19999225-233$.

9 Bennedbæk FN \& Hegedüs L. Treatment of recurrent thyroid cysts with ethanol: a randomized double-blind controlled trial. Journal of Clinical Endocrinology and Metabolism 200388 5773-5777.

10 Prapavat V, Roggan A, Walter J, Beuthan J, Klingbeil U \& Muller G. In vitro studies and computer simulations to assess the use of a diode laser $(850 \mathrm{~nm})$ for laser-induced thermotherapy (LITT). Lasers in Surgery and Medicine 199618 22-33.

11 Guglielmi R, Pacella CM, Dottorini ME, Bizzarri GC, Todino V, Crescenzi A, Rinaldi R, Panunzi C, Rossi Z, Colombo L \& Papini E. Severe thyrotoxicosis due to hyperfunctioning liver metastasis from follicular carcinoma: treatment with (131)I and interstitial laser ablation. Thyroid 1999 9 173-177.

12 Amin Z, Harries SA, Lees WR \& Bown SG. Interstitial tumour photocoagulation. Endoscopic Surgery Allied and Technology 1993 $1224-229$.

13 Brookes JA, Lees WR \& Bown SG. Interstitial laser photocoagulation for the treatment of lung cancer. American Journal of Roentgenology $1997 \mathbf{1 6 8} 357-358$.

14 Giorgio A, Tarantino L, de Stefano G, Farella N, Catalano O, Cusati B, del Viscovo L, Alaia A \& Caturelli E. Interstitial laser photocoagulation under ultrasound guidance of liver tumors: results in 104 treated patients. European Journal of Ultrasound $200011181-188$.

15 Bennedbæk FN, Karstrup S \& Hegedüs L. Ultrasound guided laser ablation of a parathyroid adenoma. British Journal of Radiology 200174 905-907.

16 Pacella CM, Bizzarri G, Guglielmi R, Anelli V, Bianchini A, Crescenzi A, Pacella S \& Papini E. Thyroid tissue: US-guided percutaneous interstitial laser ablation - a feasibility study. Radiology $2000217673-677$.

17 Law P, Gedroyc WM \& Regan L. Magnetic resonance-guided percutaneous laser ablation of uterine fibroids. Journal of Magnetic Resonance and Imaging 200012 565-570.

18 Døssing H, Bennedbæk FN \& Hegedüs L. Ultrasound guided interstitial laser photocoagulation of an autonomous thyroid nodule The introduction of a novel alternative. Thyroid $2003 \mathbf{1 3}$ 885-888.

19 Pacella CM, Bizzarri G, Spiezia S, Bianchini A, Guglielmi R, Crescenzi A, Pacella S, Toscano V \& Papini E. Thyroid tissue: US-guided percutaneous laser thermal ablation. Radiology 2004 $232272-280$.

20 Spiezia S, Vitale G, Di Somma C, Assanti AP, Ciccarelli A, Lombardi G \& Colao A. Ultrasound-guided laser thermal ablation in the treatment of autonomous hyperfunctioning thyroid nodules and compressive nontoxic nodular goiter. Thyroid 2003 13 941-947.

21 Papini E, Guglielmi R, Bizzarri G \& Pacella CM. Ultrasound-guided laser thermal ablation for treatment of benign thyroid nodules. Endocrine Practice $200410276-283$.

22 Caraccio N, Goletti O, Lippolis PV, Casolaro A, Cavina E, Miccoli P \& Monzani F. Is percutaneous ethanol injection a useful alternative for the treatment of the cold benign thyroid nodule? Five years' experience. Thyroid 19977 699-704.

23 Zingrillo M, Collura D, Ghiggi MR, Nirchio V \& Trischitta V. Treatment of large cold benign thyroid nodules not eligible for surgery with percutaneous ethanol injection. Journal of Clinical Endocrinology and Metabolism $1998 \mathbf{8 3} 3905-3907$.

24 Livraghi T, Goldberg SN, Lazzaroni S, Meloni F, Ierace T, Solbiati L \& Gazelle GS. Hepatocellular carcinoma: radio-frequency ablation of medium and large lesions. Radiology $2000 \mathbf{2 1 4} 761-768$.

Received 23 September 2004 Accepted 14 December 2004 